\title{
Diseño de un modelo de migración a cloud computing para entidades públicas de salud
}

\section{Design of a cloud computing migration \\ model for public health entreprises}

\author{
César Augusto Gutiérrez Rodríguez ${ }^{1}$, Ricardo Andrés Almeida², Wilson Eduardo Romero \\ Palacios $^{3}$
}

\section{Resumen}

Objetivo:Diseñar un modelo Cloud Computing que permitirá a las empresas manejar la información, reducir costos, maximizar servicios y utilizar tecnología de punta.

Palabras

Claves:

Computación en la nube, seguridad informática, tecnología, modelo de computación en la nube, infraestructura.

\section{Keywords:}

Cloud computing informatics security,

technology, cloud computing model, infrastructure.

Metodología: construir un modelo que genere rompimiento en el paradigma mental de los directores de las áreas de TI mediante una investigación descriptiva, realizando una observación al personal que trabaja en TI.

Resultados: se diseñó un modelo de migración basado en estrategias de diferentes autores y en la experiencia de un grupo de especialistas de un Data Center que podría servir como guía para futuras implementaciones en los hospitales de II nivel del departamento del Valle del Cauca.

Conclusiones: el modelo permitirá a los hospitales realizar la migración de su área de TI a un Data Center, alineándose con las políticas gubernamentales y ubicarse a la vanguardia en tecnología y estar a la par de multinacionales que poseen grandes recursos económicos.

\begin{abstract}
Objetive:Design a Cloud Computing model that will allow companies to manage information, reduce costs, maximize services and use state-of-the-art technology.

Methodology: to build a model that generates break in the mental paradigm of the directors of the IT areas through a descriptive investigation, making an observation to the personnel that works in IT.

Results: a migration model was designed based on strategies of different authors and on the experience of a group of specialists from a Data Center that could serve as a guide for future implementations in the II level hospitals of the department of Valle del Cauca.

Conclusions: the model will allow hospitals to migrate their IT area to a Data Center, aligning with government policies and be at the forefront of technology and be on a par with multinationals that have large economic resources.

1Corporación Universitaria Centro Superior UNICUCES ,Correo electronico: dtcis@unicuces.edu.co

2 Corporación Universitaria Centro Superior UNICUCES, Correo electronico: dirsistemas@unicuces.edu.co

3 Autor de Correspondencia, Corporación Universitaria Centro Superior UNICUCES, Correo electronico: viceacademica@unicuces. edu.co
\end{abstract} cloud computing para entidades públicas de salud", Investigación e Innovación en Ingenierias, vol. 6, n . 1, pp. 10 - 26., 2018. DOI: https://10.17081/invinno.6.1.2772 


\section{Introducción}

En el sector público colombiano la apropiación de tecnologías suele ser anticuada y muchas veces alejada de soluciones efectivas. Esto se evidencia principalmente cuando se compara con soluciones empleadas en otras partes del mundo.

Según Carsten Bruhn: "Parece que el sector público no está maximizando los beneficios del Cloud Computing, como un acceso más fácil a la información, un mayor intercambio de conocimientos y una mejor agilidad de los negocios. Esto puede que se deba a que se cuenta con procesos de documentos desconectados, en los que el sistema, la tecnología y la forma de trabajar de las personas se ven de forma aislada; en vez de ser considerados partes iguales que necesitan trabajar de forma perfectamente integrada" [1].

En el caso de Colombia, las instituciones públicas invierten anualmente una gran cantidad de recursos en tecnología, mediante inversiones que están sujetas al conocimiento y necesidades particulares de cada director de TI, quienes muchas veces evidencian la falta de pericia y de conocimiento a la hora de seleccionar la tecnología más eficiente para el modelo de negocio y para las necesidades que se requiere solucionar.

Concretamente, en el sector salud existe una gran cantidad de tecnologías diversas, que se utilizan en los hospitales; y que constituyen inversiones en tecnología que muchas veces no se analizan a profundidad. En este sentido es clara la necesidad de establecer modelos de unificación que permitan que la información de cada una de las instituciones se pueda estandarizar en un solo esquema o modelo.

"La información se convierte, entonces, en un insumo fundamental para la toma de decisiones, la orientación de políticas públicas, la formulación de estrategias, planes y programas que redunden en el bienestar de la población estudiada. Sin embargo, para que la información sea un recurso capaz de satisfacer las expectativas de los tomadores de decisiones, debe garantizarse la calidad -esto es, la confiabilidad y validez- de los datos obtenidos y el conocimiento que de ellos se infiere.

Los avances actuales en el campo de la informática aumentan cada día la probabilidad de lograr la excelencia operacional en las actividades de recolección, procesamiento, almacenamiento y comunicación de datos para la generación de información, y su automatización contribuye en gran medida a garantizar la calidad" [2].

Teniendo en cuenta todo lo anterior y en busca de una opción de mejora, la pregunta problema articulada a este proyecto es:

¿Cómo formular una estrategia para el mejoramiento de la gestión de Tecnología e Información-TI basada en las mejores prácticas del uso de Cloud Computing, a fin de obtener un aprovechamiento óptimo de los recursos, conocimiento y talento humano en estas áreas? 


\section{Metodología}

Este proyecto constituye una investigación descriptiva con un enfoque cualitativo, debido a que mediante ella se realizó una observación al personal que trabaja en TI y, con base en el resultado de las mismas, se construyó un modelo que genera un rompimiento en el paradigma mental de los directores de las áreas de TI. El estudio es de tipo exploratorio y experimental, pues el modelo propuesto servirá como guía para implementarlo teóricamente en un caso de estudio.

Por otra parte, como la propuesta que se presenta está orientada a instituciones públicas (Hospitales de II nivel de complejidad del departamento del Valle del Cauca), el diseño de esta propuesta está alineada al modelo de Gestión de Tecnologías de la Información (IT4+), presentado en el año 2016 por el Ministerio de las Tics para la gestión de las áreas de TI de las instituciones públicas de todo el país.

Básicamente, este modelo ese define de la siguiente forma:

“IT4+® es un modelo integral de gestión estratégica con tecnología cuya base fundamental es la alineación entre la gestión de tecnología y la estrategia sectorial o institucional. El modelo facilita el desarrollo de una gestión de TI que genera valor estratégico para el sector, la entidad, sus clientes de información y usuarios.

Está conformado por los siguientes componentes: Estrategia de TI, Gobierno de TI, Análisis de información, Sistemas de información, Gestión de servicios tecnológicos, Apropiación y uso." [3]

Como consecuencia de lo anterior, el modelo propuesto por los autores de este trabajo incluye los principios definidos por el MinTIC respecto al dominio de servicios tecnológicos para la arquitectura empresarial del Estado Colombiano entregado en el documento conocido como IT4+, que se recogen en la Tabla 1: 


\begin{tabular}{|c|c|c|c|}
\hline & No. & PRINCIPIO & DESCRIPCIÓN \\
\hline & 1 & Capacidad & $\begin{array}{l}\text { Hace referencia a las previsiones sobre } \\
\text { necesidades futuras basadas en tenden- } \\
\text { cias, previsiones de negocio y acuerdos } \\
\text { de nivel de servicio (ANS) existentes. }\end{array}$ \\
\hline & 2 & Disponibilidad & $\begin{array}{l}\text { Comprende la optimización y monito- } \\
\text { rización de los servicios de TI para que } \\
\text { estos funcionen ininterrumpidamente } \\
\text { y de manera fiable, cumpliendo así los } \\
\text { ANS. }\end{array}$ \\
\hline & 3 & Adaptabilidad & $\begin{array}{l}\text { De acuerdo con este principio, las im- } \\
\text { plementaciones tecnológicas deben ser } \\
\text { adaptables a las necesidades de redefini- } \\
\text { ciones en las funciones de negocio de las } \\
\text { entidades. }\end{array}$ \\
\hline $\begin{array}{r}\text { Tabla } 1 . \\
\text { Principios de la }\end{array}$ & 4 & $\begin{array}{l}\text { Cumplimiento } \\
\text { de estándares }\end{array}$ & $\begin{array}{l}\text { Toda institución del Estado cumplirá } \\
\text { como mínimo los estándares definidos } \\
\text { en la arquitectura IT4+. }\end{array}$ \\
\hline Servicios Tecnológicos & 5 & $\begin{array}{l}\text { Oportunidad en } \\
\text { la prestación de } \\
\text { los servicios }\end{array}$ & $\begin{array}{l}\text { Consiste en permitir y prestar un sopor- } \\
\text { te técnico específico de manera oportu- } \\
\text { na y efectiva. }\end{array}$ \\
\hline
\end{tabular}

IT4+ presenta entonces un conjunto de conceptos y prácticas para la gestión de servicios de tecnologías de la información, que pueden brindar descripciones detalladas de procedimientos de gestión, ideados para ayudar a las organizaciones a lograr calidad y eficiencia en las operaciones de TI. En este caso de tercerización de procesos de TI en cualquiera de sus enfoques, ya sea de infraestructura, desarrollo y operaciones.

$\mathrm{Al}$ analizar estos procesos, se puede identificar que el método de gestión puede variar, pero la responsabilidad no. En muchos casos, la calidad de la gestión interna en realidad puede resultar más crítica, pues, el hecho de que una función sea entregada al exterior, requiere de estructuras de gestión más rigurosas y disciplinadas que al interior. Hay un número importante de aspectos que requieren atención para que la aplicación de las disciplinas de IT pueda aportar beneficios significativos.

Los temas de ITIL que pueden brindar un aporte a esta decisión de tercerizar son: La Gestión de Problemas, Gestión del Cambio, Gestión de Nivel de Servicio y Gestión de la Continuidad.

En especial, cierta disciplina ITIL resulta prioritaria en este modelo: la Service Level Management. Las 
otras se emplean de forma más activa durante la transición del outsourcing. Sin embargo, el uso de ITIL ayuda sin duda a asegurar un sistema más riguroso y robusto, que en última instancia debe beneficiar a ambas partes.

Existe, además, una herramienta que se utiliza específicamente en las disciplinas de ITIL para ayudar a las organizaciones a gestionar su régimen de subcontratación inicial y permanente: El Outsourcing de IT Toolkit, que no es una simple variante del conocido kit de herramientas de ITIL, sino que incluye los componentes y documentos para ayudar a los responsables de la operación de externalización.

En este Toolkit se incluye: un acuerdo de nivel de servicio pre-escrito, un equipo de auditoría de referencia, un contrato de modelo de externalización y una guía de plan de transición de ITIL [4].

Ahora bien, el Service Level Managemenent (SLM) es la herramienta propicia para negociar, proporcionar los acuerdos de niveles de servicio y documentos con objetivos adecuados de servicio representantes de la empresa, como también la monitorización y producción de informes sobre la capacidad del proveedor de servicios para ofrecer el acuerdo de nivel de servicio (The Stationery Office, 2007). SLM es por ello un proceso vital para todos los contratantes y proveedores de servicios de TI, ya que se encarga de acordar y documentar los objetivos de nivel de servicio y responsabilidades en el Nivel de Servicio (SLA) y los Requisitos del Nivel de Servicio (SLR) para cada actividad del área involucrada.

Si estos objetivos son adecuados y reflejan con precisión los requisitos de la empresa, los servicios entregados por los proveedores de servicios se alinearán con los requisitos de la empresa y se podrá cumplir con las expectativas de los clientes y los usuarios en términos de calidad de servicio. 


\section{Resultados \\ Diseño del modelo}

En la actualidad, no existe una metodología definida o un estándar internacional aceptado para que las organizaciones adopten el modelo de computación en la nube para la operación de sus infraestructuras de TI [5]. Sin embargo, algunos autores plantean recomendaciones para que las empresas lleguen inicialmente al modelo de nube privada o híbrida y, potencialmente, pasen luego a la nube pública [6].

Cada organización define los requerimientos de integración y estandarización de sus plataformas y servicios de TI según la visión que tengan sobre su crecimiento [7]. Así, para la migración de servicios al modelo de nube, existen las llamadas "estrategias de migración", métodos que son específicos para cada solución, ya que en muchas ocasiones cada migración utiliza una metodología propia basada en unos requerimientos específicos, que difícilmente se pueden utilizar en otra migración.

Con base en estas afirmaciones y teniendo en cuenta el concepto de estrategia como: "el grupo de acciones diseñado para alcanzar una meta" [8], se sugiere la utilización del modelo de migración propuesto en el presente documento, modelo que incluye solamente hospitales de II nivel, lo que permite estandarizar las necesidades y requerimientos de estas instituciones dado que sus esquemas tecnológicos son similares.

En concreto, el modelo de migración propuesto en este documento está diseñado en cinco fases y cada fase tiene unas etapas. Este diseño se basó en la recopilación y agrupamiento de diferentes estrategias de migración propuestas en diversos documentos y en las mejores prácticas y recomendaciones dadas por ingenieros expertos en el proceso de migración al Cloud. El modelo se construyó de la siguiente manera: 


\begin{tabular}{|l|l|}
\hline ETAPA & DOCUMENTO ORIENTADOR \\
\hline ANÁLISIS & $\begin{array}{l}\text { Por Microsoft: “Cómputo en la Nube": nuevo } \\
\text { detonador para la competitividad de México. } \\
2014 \\
\text { Por IBM Cloud en su documento: “Creación } \\
\text { de una estrategia de Cloud computign”. 2014. }\end{array}$ \\
\hline PLANEACIÓN & $\begin{array}{l}\text { Por IBM Cloud en su documento: “Creación } \\
\text { de una estrategia de Cloud computign”. 2014. } \\
\text { Por IBM Cloud en su documento: “Definir un } \\
\text { ecosistema Cloud”. 2014. } \\
\text { Por Luis Joyanes Aguilar en su libro Compu- } \\
\text { tación en la nube, Estrategias del Cloud en las } \\
\text { empresas. 2012 }\end{array}$ \\
\hline DISEÑO & $\begin{array}{l}\text { Por Agencia Española de Protección de Datos } \\
\text { en su documento: “Guía para clientes que con- } \\
\text { traten servicios de Cloud Computing". 2013. } \\
\text { Por Jelle Frank van der Zwet e Ian Murphy en } \\
\text { su documento ¿Cómo migrar al Cloud?. 2015. }\end{array}$ \\
\hline EJECUCIÓN & $\begin{array}{l}\text { Infopartner, empresa para gestión de Data } \\
\text { Center. Trabajo de posgrado. Universidad } \\
\text { EAN. Bogotá. }\end{array}$ \\
\hline MONITOREO & $\begin{array}{l}\text { Por la firma consultora Gartner en su do- } \\
\text { cumento: Cloud Computing. Key Inititative } \\
\text { Overview. 2010. }\end{array}$ \\
\hline & \\
\hline
\end{tabular}

Tabla 2.

Referencias

Cabe recalcar que cada uno de los documentos mencionados anteriormente sirvieron como soporte en el diseño del modelo de migración, junto con la colaboración de los ingenieros expertos en migración y la contribución de los autores del trabajo en cada una de las etapas.

El modelo se ilustra de la siguiente manera (Figura 1): 

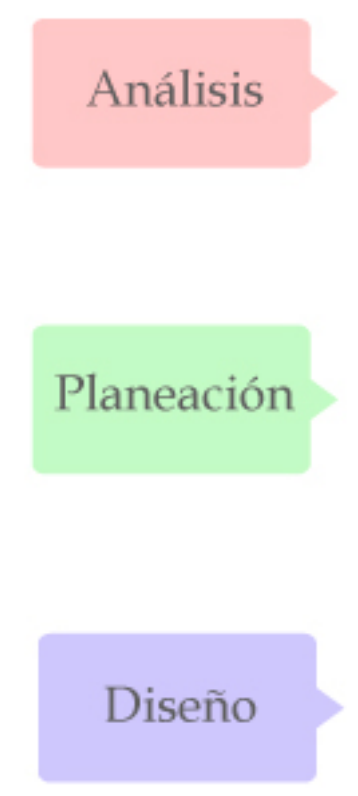

Figura 1. Principios de la Arquitectura para Servicios Tecnológicos

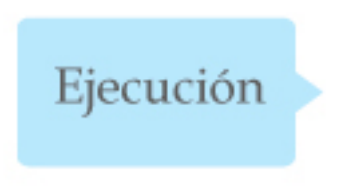

\section{Monitoreo}

- Determinar cómo el Cloud Computing ha de servir para

- lograr los objetivos del negocio y los objetivos estrategicos.

- Examinar cómo las oportunidades del Cloud Comuting se pueden implementar para incrementar el valor del negocio.

- Socialización del proyecto con la alta gerencia.

- Capacitación personal del área de Tl en el modelo Cloud.

- Análisis financiero.

- Revisión de la infraestructura de hardware y software del centro de datos.

- Análisis de la conectividad de la empresa.

- Determinar cuáles cargas de trabajo (aplicaciones) resultan más adecuadas para el despligue Cloud según su nivel de criticidad.

- Construir la estrategia de migración.

- Selección del tipo de servicio a utilizar.(SaaS, PaaS, IaaS)

- Selección del tipo de modelo a usar.(Nube ]Publica, Privada, Hibrida)

- Selección del Data Center.

- Construcción de Acuerdo SLA.

- Diseno del cronograma de migración.

- Diseno de red de datos y conectividad propuesta.

- Diseno del entorno de virtualización y recursos requeridos en el Data Center.

- Adecuación de la red de datos y conectividad.

- Migración de las cargas de trabajo según estrategia de Migración

- Pruebas de eficiencia

- Gestión y medición del servicio recibido.

\section{Análisis}

Determinar cómo el Cloud Computing va a servir para lograr los objetivos del negocio y los objetivos estratégicos. Antes de realizar cualquier tipo de migración es importante saber si la implementación de esta tecnología sirve para la organización, razón por la cual se deben revisar tanto los objetivos del negocio como los objetivos estratégicos que se encuentran descritos en el Plan Estrátegico y de esta manera determinar si utilizando la tecnología Cloud se pueden alcanzar estos objetivos.

Examinar como las oportunidades del Cloud Computing se pueden implementar para incrementar el valor del negocio. El siguiente paso lógico consiste en examinar si las oportunidades en el Cloud pueden incrementar el valor del negocio. Descubrir estas oportunidades permite ver el camino y determinar cómo alinear mejor las capacidades del Cloud en procura de los resultados esperados.

“Los proyectos de cambio tecnológico tienen una duración promedio de seis meses, desde que se 
conciben hasta que están funcionando", afirma David Alejandro Londoño, director de mercadeo de Telmex Colombia. "En computación en la nube, este tiempo se reduce a uno o dos meses, pues, como la solución ya está implementada, la única demora está en la conexión del servicio" [9].

Socialización del proyecto con la alta gerencia. Actualmente, dentro de las empresas, se ha incrementado la iniciativa de generación de proyectos en todas las áreas. A nivel mundial, "el índice de fracaso de los proyectos supera el $60 \%$, específicamente, en el ámbito TI sólo el $32 \%$ de los proyectos son exitosos, el resto resulta cuestionado por problemas $(24 \%)$ o de lleno un fracaso (44\%). Este alto porcentaje de fallas se explica porque para medir su éxito se utiliza una métrica dura y estricta". Así lo señala Sergio López, fundador y gerente general de ProactiveOffice, además de director de la Asociación de Emprendedores de Chile. El mismo López plantea que: "Un proyecto exitoso es aquél que logra un control total sobre tres dimensiones fundamentales e interdependientes: el plazo relacionado, el alcance o la calidad del producto y el presupuesto".

Evidentemente el apoyo y conocimiento de la alta gerencia es importante y vital para el desarrollo de este nuevo proyecto, razón por la cual todo el desarrollo del mismo deberá estar acompañado de los directivos y su apoyo debe ser incondicional en cada una de las etapas del mismo. En este sentido, la presentación del proyecto de migración no solo se debe exponer en términos tecnológicos y de conceptos técnicos, sino que en la exposición se debe tener una visión mucho más holística del proceso, que abarque en sí los diferentes aspectos del proyecto y muestre los diversos beneficios como son: beneficios técnicos, beneficios humanos, beneficios en infraestructura, beneficios en costos, beneficios económicos, beneficios en desempeño de los servicios, beneficios hacia los clientes. Todos estos beneficios son lo que validarán el proceso y la aceptación de la junta administrativa o el gerente de la empresa. Igualmente, se deben entregar los resultados obtenidos.

Capacitación personal del área de TI en el modelo Cloud. Uno de los recursos más importantes con los que se cuenta para la implementación de este proyecto es el personal de TI. Su conocimiento del proyecto es evidentemente necesario y obligatorio, ya que sobre ellos recae gran parte del trabajo que se necesita. En consecuencia, se le debe capacitar sobre esta nueva tecnología y sobre el desarrollo del proyecto, para que conozca lo que se pretende y asuman sus funciones en el nuevo modelo propuesto.

Análisis financiero. Como todo proyecto se debe realizar una comparación entre los gastos actuales del área de TI y los gastos al migrar esos servicios al servicio en la nube, para determinar la viabilidad financiera del proyecto.

\section{Planeación}

Revisión de infraestructura de hardware y software del centro de datos. En esta etapa del modelo se debe realizar un inventario de cada uno de los componentes de hardware, equipos de conectividad y descripción de los servidores de que se dispone. Además, se debe realizar un inventario del software con el que se cuenta en el centro de datos de la institución, revisando en concreto las licencias, el tipo de licenciamiento y fecha de caducidad. 
Análisis de la conectividad de la empresa. Ya que la columna vertebral del proceso de migración al Cloud Computing requiere de una gran conectividad, es importante que la empresa evalúe el estado de conectividad con el cual se cuenta. En específico se debe evaluar:

- La conectividad de la infraestructura interna de la empresa.

- El cableado interno de la empresa.

- Los equipos de conectividad y velocidad de los mismos.

- El estado del backbone de la empresa.

- La conectividad hacia el exterior de la empresa. (Cantidad y velocidad).

La realización de este análisis permitirá encontrar posibles fallas o rupturas que se puedan generar en la implementación de los servicios de Cloud Computing.

Clasificación de las cargas de trabajo (aplicaciones) por niveles de criticidad para ser migradas. El plan de continuidad del negocio establece los procedimientos que aplica una organización en caso de una contingencia grave o un desastre. En dicho plan se incluye el plan de continuidad de servicios de TI (en inglés: IT service continuity, ITSC), en el cual se ha de determinar la forma en que los servicios de TI pueden apoyar o soportar el negocio en caso de interrupción.

Para realizar la identificación de los servicios críticos, se utilizó una herramienta metodológica utilizada en la Migración de los servicios de la Aerocivil al modelo en Nube. Esta, herramienta fue diseñada por los ingenieros más experimentados en temas de tecnología de información de dicha empresa.

Esta evaluación fue realizada de acuerdo con la clasificación de las amenazas, los criterios de probabilidad de ocurrencia, el impacto en caso de falla, la tolerancia al riesgo y el número de usuarios afectados. Esta clasificación se definió de acuerdo con la Tabla 2.

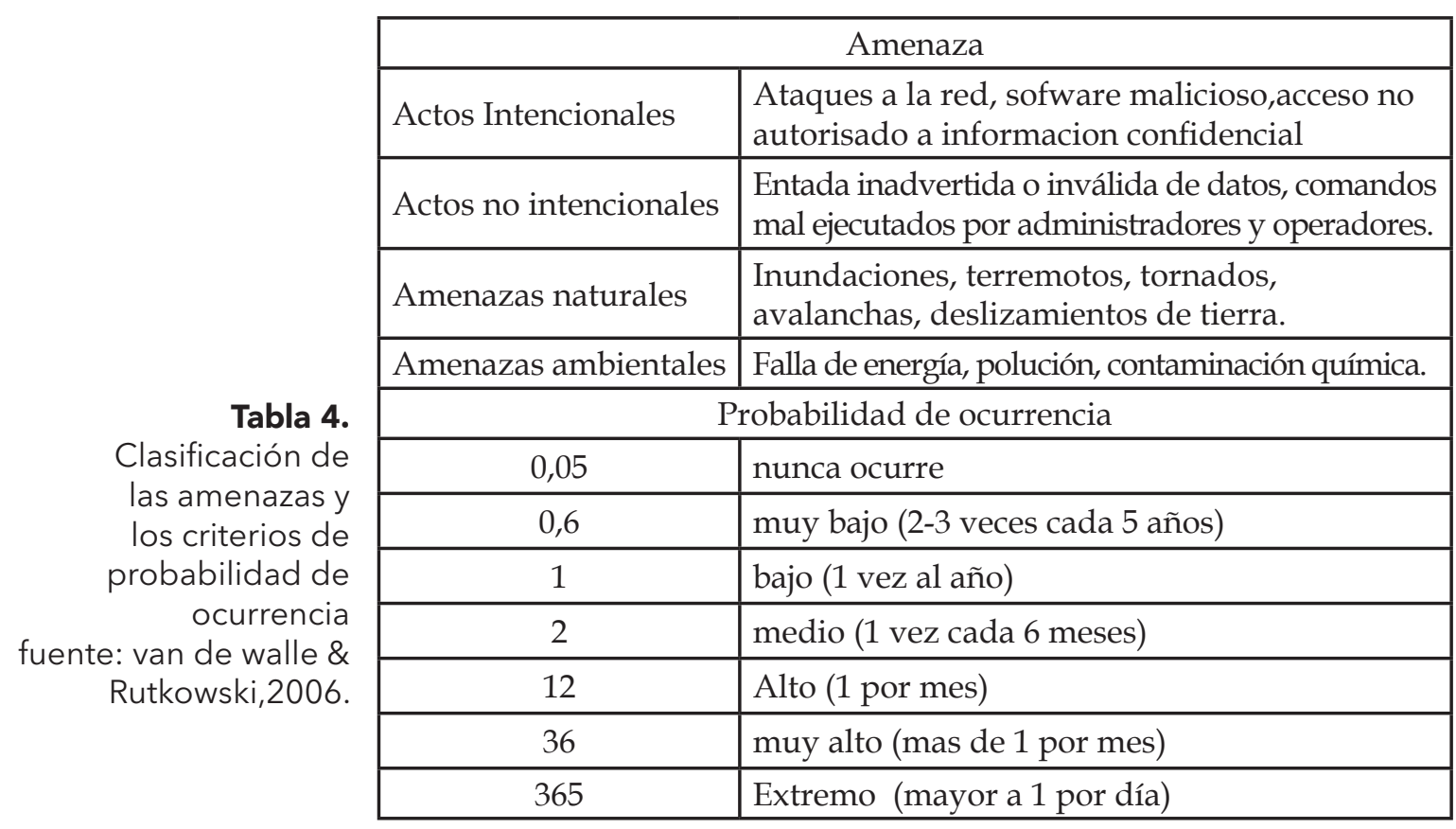


La tolerancia al riesgo (Tabla 3), definida como el número de horas en que la organización puede operar sin el servicio de TI sin que se afecte la prestación de los servicios, y los Niveles de impacto (Tabla 4) permite evaluar el daño colateral que genera la caída del servicio.

\begin{tabular}{|c|l|}
\hline $\begin{array}{c}\text { Tolerancia al riesgo } \\
\text { (hora) }\end{array}$ & \multicolumn{1}{c|}{ Descripción } \\
\hline 4 & $\begin{array}{l}\text { Sistemas de información misionales y servicios de } \\
\text { TI de apoyo a toda la organización. }\end{array}$ \\
\hline 24 & $\begin{array}{l}\text { Sistemas de información de apoyo a las labores } \\
\text { administrativas y servicios de administración } \\
\text { y gestión de TI. }\end{array}$ \\
\hline 72 & $\begin{array}{l}\text { Sistemas de información y servicios de TI de } \\
\text { apoyo sólo a procesos específicos. }\end{array}$ \\
\hline
\end{tabular}

Tabla 5.

Clasificación de la tolerancia al riesgo fuente (10)

\begin{tabular}{|c|l|}
\hline $\begin{array}{c}\text { Tolerancia al riesgo } \\
\text { (hora) }\end{array}$ & \multicolumn{1}{c|}{ Descripción } \\
\hline 10 & $\begin{array}{l}\text { Bajo: no afecta la misión de una organización, la } \\
\text { reputación o los intereses. }\end{array}$ \\
\hline 50 & $\begin{array}{l}\text { Medio: afecta notablemente la misión de una } \\
\text { organización, la reputación o los intereses. }\end{array}$ \\
\hline 100 & $\begin{array}{l}\text { Alto: impide la misión de una organización, la } \\
\text { reputación o los interese. }\end{array}$ \\
\hline
\end{tabular}

Tabla 6.

Clasificación de la tolerancia al riesgo fuente (10)

La criticidad para los productos y servicios de TI que administra la Dirección de Informática se presenta en la Tabla 5, en la cual están ordenados por el índice de criticidad, la tolerancia al riesgo y el número de usuarios afectados.

\begin{tabular}{|c|c|c|c|c|c|c|c|c|}
\hline No & $\begin{array}{c}\text { Servicio } \\
\text { de TI }\end{array}$ & Amenaza & $\begin{array}{l}\text { Probabilidad } \\
\text { de ocurrencia }\end{array}$ & Impacto & $\begin{array}{l}\text { Índice de } \\
\text { Criticidad }\end{array}$ & $\begin{array}{l}\text { Tolerancia } \\
\text { al Riesgo }\end{array}$ & $\begin{array}{l}\text { Usuarios } \\
\text { afectados }\end{array}$ & \multirow{5}{*}{$\begin{array}{l}\text { Tabla } 7 . \\
\text { Criticidad de } \\
\text { los productos } \\
\text { y servicios } \\
\text { de TI } \\
\text { elaboración } \\
\text { propia. }\end{array}$} \\
\hline \multirow{4}{*}{1} & \multirow{4}{*}{ Servicio } & $\begin{array}{l}\text { Actos } \\
\text { intencionales }\end{array}$ & & & & & & \\
\hline & & $\begin{array}{l}\text { Actos no } \\
\text { intencionales }\end{array}$ & & & & & & \\
\hline & & $\begin{array}{l}\text { Desastres } \\
\text { naturales }\end{array}$ & & & & & & \\
\hline & & $\begin{array}{l}\text { Riesgos } \\
\text { ambientales }\end{array}$ & & & & & & \\
\hline
\end{tabular}


La anterior tabla se utiliza de la siguiente manera:

El director de TI de la institución según su conocimiento de la institución, deberá:

1. Asignar un valor para Probabilidad de ocurrencia

2. Asignar un valor de Impacto

3. Calcular el índice de criticidad con el valor promedio de la probabilidad de ocurrencia de cada amenaza, multiplicado por el impacto.

4. Asignar un valor para tolerancia al riesgo

5. Asignar un valor al número de usuarios afectados en caso de falla del servicio.

Los servicios de TI con mayor índice de criticidad, con menor número de horas de tolerancia al riesgo y con mayor número de usuarios afectados son los más críticos y, por tanto, deben migrarse.

Construir la estrategia de migración. En esta etapa se debe planificar como se va a realizar la migración de cada uno de los servicios, dependiendo de las características de cada aplicación, su nivel de criticidad y el estado actual de la infraestructura física de la institución.

Selección del tipo de servicio a utilizar (SaaS, PaaS, IaaS). Una vez se tiene claro el tipo de infraestructura con la que se cuenta se deben seleccionar los tipos se servicios Cloud a contratar: SaaS: Software que se desea arrendar, que se requieren actualizar, que se quieren estandarizar. IaaS: Equipos, servidores y toda la infraestructura que se desea arrendar en el Data Center. (En este punto es válido hacer uso del servicio de Colocación en el Data Center).

PaaS. Alquiler de una plataforma de desarrollo para la construcción y puesta en marcha de aplicaciones propias y servicios Web que estarán completamente disponibles a través de Internet.

Selección del tipo de modelo a usar (Nube Pública, Privada, Hibrida). En esta etapa se debe definir el tipo de nube a utilizar (privada, pública o hibrida), dependiendo del servicio y el nivel de seguridad que se requiere.

\section{Diseño}

Selección del Data Center. Para la selección del Data Center más óptimo a la solución planteada, es importante que en el diseño se estudien todas las características que ofrece c/u de los Data Center que prestan sus servicios en Colombia. Enseguida se presenta una breve descripción de las características que se deben buscar.

- Que cuente con certificaciones recientes y actualizadas (TIER, ISO, CCNA, norma TIA 942).

- Que posea Soporte 24×7×364.

- Área de trabajo y de pruebas de migración como servicio incluido.

- Formación y certificaciones del personal de TI del Data Center.

- Identificar cuáles son sus enlaces redundantes de salida a internet.

- Ubicación geográfica.

- SolideZ presupuestal del Data Center. 
Acompañamiento en la migración.

Disponibilidad para auditorías y revisiones por parte de terceros.

Aspectos como: energía, enfriamiento, seguridad, reportes e informes, atención al cliente, tecnología y costos de los servicios.

En el mercado colombiano ya existe un gran número de empresas dedicadas al negocio de servicios en la nube, lo que facilita realizar comparaciones para determinar cuáles ofrecen las mejores alternativas para los servicios requeridos.

Existen varios criterios para comparar y seleccionar proveedores [11], entre los que se destacan:

- Número de años en el negocio

- Estabilidad financiera

- Capacidad técnica

- Calidad del desempeño pasado

- Capacidad de dirección de proyectos recios

El proveedor de servicios debe ofrecer todas las garantías y experiencia para que la organización pueda confiar la operación de sus servicios informáticos y la custodia de su información [11].

Construcción de acuerdo SLA. Uno de los aspectos más importantes de este proceso son las responsabilidades que adquieren tanto la empresa como el proveedor del servicio de Data Center, razón por la cual las cláusulas que se de este acuerdo deben respetar las normas gubernamentales en el tratamiento de la información pública. así como Además, se deben implementar mecanismos que protejan la información a colocar en el Data Center.

Este acuerdo es claramente un elemento muy importante en la documentación para ambas partes. Si se utiliza correctamente debe contener [12,13]:

- Disponibilidad del servicio.

- Garantía del hardware arrendado

- Tiempo de respuesta a las incidencias según su nivel de criticidad.

Seguridad.

- Identificar y definir las necesidades del cliente.

- Proporcionar un marco para la comprensión.

- Simplificar cuestiones complejas.

- Reducir las áreas de conflicto.

- Ánimo de diálogo en caso de conflicto.

- Eliminar las expectativas poco realistas.

- Los servicios que se entregan.

- Rendimiento, seguimiento e informes de Gestión de Problemas.

- Cumplimiento legal.

- Deberes y responsabilidades del cliente.

- Derechos de propiedad intelectual y la información confidencial de terminación. 
Diseño del cronograma de migración. Como todo proyecto, este debe estar regulado por un cronograma de actividades y tiempos, se debe crear un cronograma macro que muestre el todo el proyecto de migración y todos los servicios que se pretenden subir a la nube y de la misma manera se deberá realizar para cada uno de los servicios un cronograma que muestre la transición del servicio al modelo en nube.

Diseño de red de datos y conectividad propuesta. Basados en el análisis de conectividad de la empresa, en el que se evidencian las posibles fallas que se pueden presentar al implementar el modelo en la nube, se debe desarrollar un diseño de la nueva red de datos interna, así como el diseño de la nueva conectividad de la infraestructura de la empresa hacia el exterior y la conectividad al Data Center.

Diseño del entorno de virtualización y recursos requeridos en el Data Center. Una vez se empiezan a definir las cargas de trabajo que se van a migrar en un orden concreto, es necesario relacionar aplicaciones con necesidades en infraestructura de hardware y software, tarea en la que se debe buscar la asesoría del Data Center.

\section{Ejecución}

Adecuación a red de datos y conectividad. En esta etapa del modelo se adquieren los equipos requeridos para una buena conexión, su configuración a la red de datos y la implementación de una buena conectividad hacia el Data Center, lo que incluye una redundancia en la salida a Internet y un firewall para la protección interna de la infraestructura en el hospital.

Migración de servicios según la estrategia de migración. En este paso se precisan las cargas de trabajo a migrar, según la estrategia de migración construida y en las etapas que se definieron previamente.

\section{Monitoreo}

Pruebas de eficiencia. En esta etapa del modelo se deben realizar las pruebas de eficiencia que permitan determinar el correcto funcionamiento de cada una de las aplicaciones migradas al Cloud. Gestión y medición del servicio recibido. La etapa final consiste en la gestión de la relación con el proveedor de servicios de Cloud, así como la verificación y medición del desempeño de cada una de las aplicaciones migradas y de los servicios entregados por el proveedor de Cloud. 


\section{Conclusión}

En los informes presentados por firmas consultoras en TI a nivel internacional, se evidencia que diferentes gobiernos y empresas privadas están utilizando al Cloud Computing como una herramienta tecnológica en sus áreas de TI.

De la misma manera, en informes como los de GARTNER, IDC y otros, se observa que las empresas privadas y públicas del mundo están haciendo más uso de servicios de TI como el Cloud y dejando en un segundo lugar la compra de hardware y software, lo que difiere de las prioridades en Latinoamérica y Colombia, donde la compra de Hardware y Software son prioridad a la hora de hacer inversiones en TI.

Por otra parte, el mercado del diseño de software en el mundo se está orientando hacia la creación de aplicaciones de pago por uso, ya que su rentabilidad es mucho mayor que la de pago único por compra de licencia. Esto ha cambiado los paradigmas de las empresas diseñadoras de software en Colombia y conlleva al uso del Cloud.

Actualmente, el Gobierno Nacional y el Ministerio de las TICS están impulsando a las instituciones públicas a ser más eficientes. En este marco. el presente modelo permitirá a los hospitales que deseen realizar la migración de su área de TI a un Data Center, alinearse con las políticas gubernamentales, ubicarse a la vanguardia en tecnología y estar a la par de multinacionales que poseen grandes recursos económicos.

Tecnologías como el Cloud Computing permitirán que instituciones públicas como los hospitales de II nivel de Colombia, y en especial los del Valle del Cauca, puedan acceder a tecnología de punta a bajos costos y a estándares internacionales de calidad, lo cual es un importante logro que se verá reflejado en la mejora del servicio y en la satisfacción de sus usuarios.

La implementación del modelo de Cloud Computing también permitirá que las áreas de TI de los hospitales se orienten más hacia los objetivos del negocio y hacia los objetivos estratégicos de la alta dirección, y menos al mantenimiento y soporte de la infraestructura, tal como se demuestra en el análisis de las encuestas.

Finalmente, el estudio permitió conocer la situación actual del área de sistemas de las instituciones públicas del sector salud de II nivel en el departamento del Valle del Cauca, revelando que existen debilidades en su centro de datos, tales como la baja seguridad en la custodia de su sistema de información y de los backup; la escasa inversión en el fortalecimiento de su infraestructura tecnológica y un atraso tecnológico evidente. Esto se debe a que la dirección de estas entidades aún piensa que las Tecnologías de Información no aportan valor real al negocio, que son muy costosas y, en síntesis, desconocen los aportes que podrían entregar estas nuevas tecnologías a su empresa. 


\section{Referencias}

1. Ricoh, El sector público lidera la adopción del cloud computing aunque desconoce cómo sacarle el máximo provecho, 2013. Recuperado el (29/06/2016) desde http://www.ricoh.es/sobre-ricoh/noticias/2013/sector-publico-cloud-computing.aspx

2. Ministerio de Protección Social - Universidad de Antioquia. (2010). Análisis de la situación de salud en Colombia 2002 - 2007.

3. Ministerio de las Tecnologías de la Información y Comunicación,(2016). Modelo de Gestión IT4+. Recuperado el (13/11/2016) Disponible en: http://estrategia.gobiernoenlinea.gov.co

4. Itil.osiatis.es. (2016). ITIL ${ }^{\oplus}$ - Gestión de Servicios TI. Recuperado el (1/11/2016), de http://itil.osiatis.es/ Curso_ITIL/

5. A. Mohammad \& H. Mcheick, Cloud services Testing: An Undesrtandig. (E. Ltda, Ed.), 2011.

6. R. Blandford, Information security in the cloud. Network security, pp. 15-17, 2011.

7. P. Weill \& J. Ross, IT Savvy. Boston: Harvard Business School Publishing, 2009.

8. Bateman \& Snell, Administración de una ventaja competitiva. México: Mc Graw-Hill, 2001.

9. Management Solutions, La nube: Oportunidades y retos para los integrantes de la cadena de valor, 2012.

Disponible en: https://www.managementsolutions.com/sites/default/files/publicaciones/esp/La-nube.pdf.

Visto el 10/03/2017

10. O.I. Páez Murcia, La computación en la nube, como solución a los problemas de disponibilidad y continuidad en los servicios informáticos de la aeronáutica civil. Maestría thesis, Universidad Nacional de Colombia, 2012.

11. PMI, Project Management Institute, 2008.

12. Service Level Agreement Zone. The Service Level Agreement, 2015. Disponible en http://www.sla-zone. co.uk/index.htm. visto el: 13/03/2017.

[13. L. Ferrer Castellanos, K. González Insignares and L. Mendoza Vega, "La innovación como factor clave para mejorar la competitividad de las pymes en el departamento del Atlántico, Colombia”, Dictamen Libre, no. 16, pp. 21-36, 2015.

\section{Bibliografía de consulta}

- IMC, Instituto Mexicano de la competitividad. "Cómputo en la nube": nuevo detonador para la competitividad de México, 2012. http://imco.org.mx/wp-content/uploads/2012/6/computo_en_la_nube_detonador_de_competitividad_doc.pdf

- NIST, National Institute Of Standards and Technology. Effectively and Securely Using de Cloud Computing paradigm, 2009. Recuperado el (29/06/2016) desde http://crsc.nist.gov/groups/sn/cloud-computing/ cloud-computing-v25.ppt

- University of California at Berkeley, Above the Clouds: A Berkeley, 2009. Recuperado el (10/05/2016) desde https://www.eecs.berkeley.edu/Pubs/TechRpts/2009/EECS-2009-28.pdf

- GARTNER. Cloud Computing - Key Initiative Overview, 2010. Recuperado el (29/06/2016) desde http:// www.gartner.com/it/initiatives/pdf/KeyInitiativeOverview_CloudComputing.pdf

Como citar este artículo: C.A. Gutiérrez, R. Almeida., y W. Romero,“Diseño de un modelo de migración a cloud computing para entidades públicas de salud", Investigación e Innovación en Ingenierias, vol. 6, n. 1, pp. 10 - 26., 2018. DOI: https://10.17081/invinno.6.1.2772 
- L. Joyanes, Computación en la Nube - Estrategias de Cloud Computing en las empresas. México: Editorial Alfaomega. Primera edición, 2012.

- G. Palacio, Data Centers Hoy - Protección y Administración de datos en la empresa. Editorial S.A. MARCOBO. Primera Edición, 2014.

- M. Guilarte, ¿Qué es un Tier?, 2013. Recuperado el (30/09/2016), de: http://www.muycomputerpro. com/2013/03/14/que-es-un-tier.

- J. Doña, J. E. García, J. López, F. Pascual, \& F. Pascual, Virtualización de Servidores-Una Solución de Futuro. Hospital Universitario Virgen de La Victoria, 2010. Málaga. España.

- M. Lopez, E. Huedo \& J. Garbajosa. GreenIT: Tecnologías para la eficiencia energética en los sistemas T.I. - Informe de Vigilancia Tecnológica, 2012.

- J. Doña, J. Garcia, J. Lopez, F. Pascual, R. Pascual. Virtualización de servidores-Una solución de Futuro. Campus Universitario de Teatinos. Málaga. España, 2010.

C. Wolf y E. M. Halter. Virtualización. From the deskopt to the Enterprise. APRESS, 2005, p. 600.

F. Rolón, "Opciones de la banca comercial en productos de importación", Dictamen Libre, no. 1213, pp. 71-75, 2013.

- H. Hernández Palma, «Sistemas de gestión integrados en el sector salud para la optimización de la calidad en el departamento del atlántico,» Dictamen Libre, no 20, pp. 99-106, 2017. 